\title{
Perirhinal cortex and feature-ambiguous discriminations
}

\author{
Timothy J. Bussey, ${ }^{1,3}$ Lisa M. Saksida, ${ }^{1}$ and Elisabeth A. Murray ${ }^{2}$ \\ ${ }^{1}$ Department of Experimental Psychology, University of Cambridge, Cambridge CB1 3EB, United Kingdom; ${ }^{2}$ Laboratory \\ of Neuropsychology, National Institute of Mental Health, NIH, Bethesda, Maryland 20892, USA
}

Perirhinal cortex is often regarded as part of a medial temporal lobe (MTL) memory system, mediating memory selectively, and having little role in other functions such as perception (e.g., Squire and Zola-Morgan 1991; Buffalo et al. 1998, 1999; Squire et al. 2004). We and others have argued against this idea, suggesting instead that perirhinal cortex may have a role in perception (e.g., Buckley and Gaffan 1997; Murray and Bussey 1999; Buckley et al. 2001; Bussey and Saksida 2002). Evidence to support this view has come from recent work indicating that lesions of perirhinal cortex can impair the discrimination of visual stimuli. Perirhinal cortex is not, however, important for all types of visual discrimination (Buckley and Gaffan 1997; Hampton and Murray 2002), only for those requiring representations of complex conjunctions of features (Saksida and Bussey 1998; Murray and Bussey 1999; Buckley et al. 2001; Eacott et al. 2001; Bussey and Saksida 2002; Bussey et al. 2002, 2003). These discriminations possess a high degree of "feature ambiguity," a property of visual discriminations that can emerge when features are a part of both rewarded and unrewarded stimuli (Bussey and Saksida 2002). In one such study (Bussey et al. 2003), we used complex featureambiguous "morphed" photographic stimuli to test this idea (Fig. 1). Monkeys were first trained on a pairwise discrimination of unmorphed photographs (e.g., images 1 and 40 in Fig.1). This was followed by the critical performance test session, in which the monkeys were presented with three pairs of stimuli: the trained pair, and two pairs in which the photographs had been morphed together to different degrees, thus creating three levels of perceptual difficulty. It was found that monkeys with perirhinal cortex lesions were impaired on the perceptually difficult, but not the perceptually easy, pairs (Fig. 2). This impairment was specific to complex, high feature-ambiguity discriminations: The lesions did not affect difficult color or size discriminations, indicating that the impairment was not due simply to the difficulty of the discriminations.

Levy et al. (2005) investigated visual discrimination ability in amnesic patients using similar stimulus materials to those used in our monkey study. In their report, these authors challenge our previously published results, and call for a reanalysis of our previously published data, suggesting that an impairment in new learning may explain the poor performance in our monkeys with perirhinal cortex damage. Specifically they ask:

"In the case of the study with blended images, it was suggested that learning was required during initial discrimination learning but not during the 32 test trials with blended images. Accordingly, it was suggested that impaired performance during the trials with blended images reflected impaired visual perception, not an impairment in learning and memory. This interpretation was based on an analysis of variance that indicated that no further learning occurred across the 32 trials (the 32 trials were

\section{${ }^{3}$ Corresponding author.}

E-mail tjb1000@cam.ac.uk; fax +44 (0) 1223333564.

Article and publication are at http://www.learnmem.org/cgi/doi/10.1101/ Im.163606. partitioned into eight four-trial blocks). There was also no group $\mathrm{x}$ trial-block interaction. Yet, one wonders how monkeys performed on the first one or two blocks of the test trials with blended images. Did generalization fail altogether? One also wonders whether the impaired performance during these test trials could reflect to some degree the fact that control monkeys improved more during the test trials than the monkeys with perirhinal lesions. Did no learning occur at all? Accordingly, it

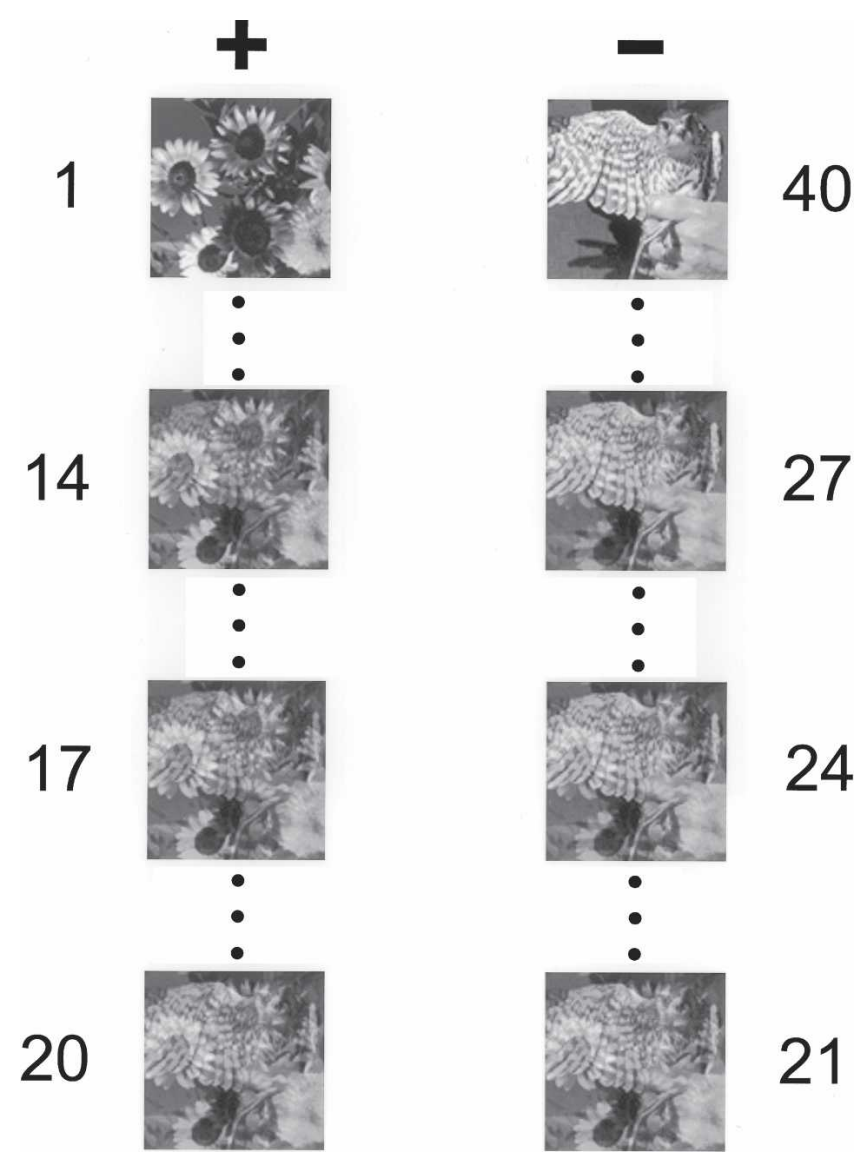

Figure 1. Example of grayscale picture stimuli used in Bussey et al. (2003). To create each pair of stimuli, two pictures were "morphed" (blended) together to create a series of 40 images, the first images in this series consisting mostly of features from picture 1 , the latter images consisting mostly of features from picture 2 . The numbers next to an image indicate the position of the image in the series. In the experiment described here (Experiment 2), the original images, numbers 1 and 40 , were used as the low feature ambiguity "trained pair," images 14 and 27 were chosen to be the high feature ambiguity "pair 1 " and images 17 and 24 were chosen to be the high feature ambiguity "pair 2." The + and - indicate that the images on the left, in this example those most similar to the photograph of the sunflowers, were the correct (rewarded) images in the pair, whereas those on the right were incorrect (unrewarded), respectively. 
would be of interest to inspect the performance curves across the blocks of trials with blended images to determine whether there was any sign of differential learning."

We are very happy to reply to this query. First, we would emphasize that we did not rely only on a lack of interaction to conclude that there was no learning. Indeed, as described in the paper, we went on to test for main effects of Block, for both the control and the lesion groups, in both of the featureambiguous conditions. As indicated in our original report, there was no significant learning across blocks in any of these four tests (all $F<1$ ). Second, the data from individual blocks are shown in Figure 3. The difference between groups at the outset of the 32 blocks is, if anything, greater than at the end. It is quite clear that this is not a learning effect.

There is, however, one caveat with respect to our study that warrants attention. It is possible that scores on the morph performance test might have been influenced by comparing the morphed S+ (correct stimulus) with memory for the trained (unmorphed) $\mathrm{S}+$ presented in the same session. Ideally one would present the trained $\mathrm{S}+$ alongside the morphed stimuli so that if such a comparison were facilitating performance, it could be made without recourse to memory. This is precisely the method used by Lee et al. (2005), who tested patients with either selective hippocampal damage, or with combined damage to hippocampus and extra-hippocampal regions predominantly in perirhinal and entorhinal cortex (the "MTL group"). The findings parallel those in our monkey study: The MTL group, and not the patients with selective hippocampal lesions, was impaired on high feature-ambiguity morph discriminations. In addition, these authors included morphed photographs of spatial scenes. Strikingly, patients with selective hippocampal lesions were selectively impaired in the visual discrimination of morphed scenes, suggesting that not only perirhinal cortex, but the hippocampus as well, may have a role in perception. Importantly, as in the reanalysis above, these impairments cannot be explained by new learning; in similar fashion to our control monkeys, control subjects in that study did not learn across testing trials, and if anything performed better at the outset of testing than they did at the end.

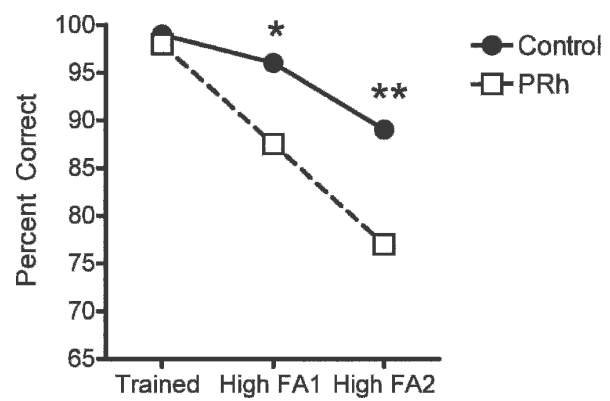

Figure 2. Discrimination performance of intact monkeys (Control) and monkeys with lesions of perirhinal cortex (PRh) on the low feature ambiguity trained pair, and on the two high feature ambiguity pairs. Monkeys were trained on four problems of the type shown in Figure 1, and performance test scores were averaged over the four problems to generate the curves shown here. Asterisks indicate significant differences between the groups; ${ }^{*} P<0.05 ;{ }^{* *} p<0.01$. Data are from Bussey et al. (2003), Experiment 2.
In sum, our reanalysis reconfirms our original interpretation, showing that-consistent with similar analyses in recent studies of human amnesic patients-an impairment in new learning cannot explain our results. (For a review of these monkey and human studies, see Bussey and Saksida 2005.)

Finally, a correction to the Levy et al. (2005) report is necessary. The authors state that our monkeys with perirhinal cortex lesions were impaired in the initial single-pair discriminations, before they were tested on the morph discriminations. This is an important issue, as such an initial impairment could affect interpretation of the subsequent morph tests. However, Levy et al.'s statement is not correct: There was in fact no such impairment $\left(F_{1,6}=3.3 ; P=0.12\right)$. Perhaps Levy et al. meant that there was a numerical difference between the group means, but of course this was not a statistically significant difference. This lack of impairment in acquisition of a single visual discrimination is supported by the results of Experiment 1 in our study (Bussey et al. 2003). This experiment showed, quite clearly, that perirhinal cortex lesions impaired slowly learned morphed discriminations and not rapidly learned ones, precisely the opposite pattern to that predicted by Squire and colleagues (Squire and Zola-Morgan 1983; Zola and Squire 2000). Thus, the critical factor was not speed of learning, but feature ambiguity.

We hope that this Letter to the Editor adequately addresses the queries posed by Levy et al. (2005). The idea that the MTL structures may have a role in perception is clearly highly controversial, and will likely generate much further experimentation and debate. The result will no doubt be a greatly enriched understanding of the organization of learning, memory, and perception in the medial temporal lobe.

\section{References}

Buckley, M.J. and Gaffan, D. 1997. Impairment of visual object-discrimination learning after perirhinal cortex ablation. Behav. Neurosci. 111: 467-475.

Buckley, M.J., Booth, M.C., Rolls, E.T., and Gaffan, D. 2001. Selective perceptual impairments after perirhinal cortex ablation. J. Neurosci. 21: 9824-9836.

Buffalo, E.A., Reber, P.J., and Squire, L.R. 1998. The human perirhinal cortex and recognition memory. Hippocampus 8: 330-339.

Buffalo, E.A., Ramus, S.J., Clark, R.E., Teng, E., Squire, L.R., and Zola, S.M. 1999. Dissociation between the effects of damage to perirhinal cortex and area TE. Learn. Mem. 6: 572-599.

Bussey, T.J. and Saksida, L.M. 2002. The organization of visual object representations: A connectionist model of effects of lesions in perirhinal cortex. Eur. J. Neurosci. 15: 355-364.

. 2005. Object memory and perception in the medial temporal lobe: An alternative approach. Curr. Opin. Neurobiol. 15: 730-737.

Bussey, T.J., Saksida, L.M., and Murray, E.A. 2002. Perirhinal cortex resolves feature ambiguity in complex visual discriminations. Eur. J. Neurosci. 15: 365-374.

\section{Learning \& Memory}

www.learnmem.org 
2003. Impairments in visual discrimination after perirhinal cortex lesions: Testing "declarative" versus "perceptual-mnemonic" views of perirhinal cortex function. Eur. J. Neurosci. 17: 649-660.

Eacott, M.J., Machin, P.E., and Gaffan, E.A. 2001. Elemental and configural visual discrimination learning following lesions to perirhinal cortex in the rat. Behav. Brain Res. 124: 55-70.

Hampton, R.R. and Murray, E.A. 2002. Stimulus representations in rhesus monkeys with perirhinal cortex lesions. Behav. Neurosci. 116: $363-377$.

Lee, A.C., Bussey, T.J., Murray, E.A., Saksida, L.M., Epstein, R.A., Kapur, N., Hodges, J.R., and Graham, K.S. 2005. Perceptual deficits in amnesia: Challenging the medial temporal lobe "mnemonic" view. Neuropsychologia 43: 1-11.

Levy, D.A., Shrager, Y., and Squire, L.R. 2005. Intact visual discrimination of complex and feature-ambiguous stimuli in the absence of perirhinal cortex. Learn. Mem. 12: 61-66.

Murray, E.A. and Bussey, T.J. 1999. Perceptual-mnemonic functions of perirhinal cortex. Trends Cogn. Sci. 3: 142-151.

Saksida, L.M. and Bussey, T.J. 1998. Toward a neural network model of visual object identification in primate inferotemporal cortex. Abstract Viewer/Itinerary Planner. Program No. 1906. Society for Neuroscience, Washington, DC.

Squire, L.R. and Zola-Morgan, S. 1983. The neurology of memory: The case for correspondence between the findings for human and nonhuman primate. In The physiological basis of memory, pp. 199-267. Academic Press, New York.

1991. The medial temporal lobe memory system. Science 253: $1380-1386$.

Squire, L.R., Stark, C.E., and Clark, R.E. 2004. The medial temporal lobe. Annu. Rev. Neurosci. 27: 279-306.

Zola, S. and Squire, L.R. 2000. The medial temporal lobe and the hippocampus. In The Oxford handbook of memory (eds. E. Tulving and F.I.M. Criak), pp. 485-500. Oxford University Press, Oxford, UK.

Received July 19, 2005; accepted in revised form December 16, 2005. 


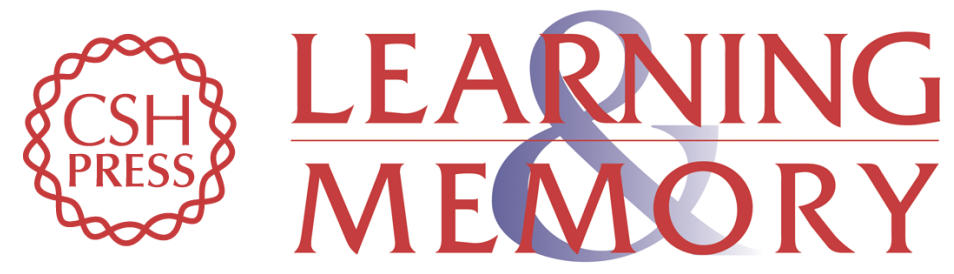

\section{Perirhinal cortex and feature-ambiguous discriminations}

Timothy J. Bussey, Lisa M. Saksida and Elisabeth A. Murray

Learn. Mem. 2006, 13:

Access the most recent version at doi:10.1101/lm.163606

References This article cites 15 articles, 4 of which can be accessed free at: http://learnmem.cshlp.org/content/13/2/103.full.html\#ref-list-1

License

Email Alerting Receive free email alerts when new articles cite this article - sign up in the box at the Service top right corner of the article or click here. 